\title{
Impact of antifungal stewardship interventions on the susceptibility of Candida species in pediatric patients with malignancy
}

\author{
Ali Amanati \\ Shiraz University of Medical Sciences \\ Parisa Badiee \\ Shiraz University of Medical Sciences \\ Hadis Jafarian \\ Shiraz University of Medical Sciences \\ Fatemeh Ghasemi \\ Shiraz University of Medical Sciences \\ Samane Nematolahi \\ Shiraz University of Medical Sciences \\ Sezaneh Haghpanah \\ Shiraz University of Medical Sciences \\ Seyedeh Sedigheh Hamzavi ( $\sim$ s.hamzavi55@yahoo.com ) \\ Shiraz University of Medical Sciences
}

\section{Research}

Keywords: Candida species, antifungal susceptibility, broth microdilution method, Hematologic Neoplasms, Antifungal stewardship program, Drug resistance, Fungal

Posted Date: August 25th, 2020

DOI: https://doi.org/10.21203/rs.3.rs-63065/v1

License: (1) This work is licensed under a Creative Commons Attribution 4.0 International License. Read Full License 


\section{Abstract \\ Background}

There is a worldwide concern with respect to the antimicrobial resistance and the inappropriate use of antifungal agents, which had led to an ever-increasing antifungal resistance. This study aimed to identify the antifungal susceptibility of Candida species isolated from the pediatric patients with cancer and also to evaluate the clinical impact of antifungal stewardship (AFS) interventions on antifungal susceptibility of Candida species.

\section{Methods}

In this study, Candida species colonization were evaluated among hospitalized children with cancer in a tertiary teaching hospital, Shiraz 2017-18. The broth microdilution method was used to determine the minimum inhibitory concentrations (MICs) for polyenes (amphotericin B), echinocandins (caspofungin), and azoles (voriconazole, fluconazole, posaconazole, and itraconazole). Antifungal susceptibility of Candida species was compared with our previous study results to determine the clinical impact of AFS interventions on the antifungal susceptibility.

\section{Results}

The prevalence of Candida albicans in the present study was significantly higher than other Candida species. Candida albicans species were completely susceptible to the azoles. The sensitivity rate of $C$. albicans to amphotericin B and caspofungin was $93.1 \%$ and $97.1 \%$, respectively. The results confirm the positive effect of optimized antifungal usage and bedside intervention on the susceptibility of Candida species after the implementation of the AFS program. C. albicans and $C$. glabrata exhibited significant increase in sensitivity after the execution of AFS program.

\section{Conclusions}

Improving the antifungal agent usage can improve antifungal susceptibility and reduce resistance. The AFS is recommended to be addressed, applied, and regularly assessed in centers.

\section{Introduction}

The prevalence of Candida infections is on the rise as a result of excessive usage of broad-spectrum antibiotics, HIV infection, malignancies, transplants, invasive procedures, and prolonged hospitalization [1-3]. More than 17 different species of Candida are recognized that can infect humans, and amongst them, $90 \%$ of all invasive infections are related to C. albicans, C. glabrata, C. parapsilosis, C. tropicalis, and C. krusei $[4,5]$.

Antifungal resistance usually occurs following selective pressure induced by the use or misuse of antifungal agents in high-risk patients, especially those with malignancy [6-8]. Candida infections epidemiology changes by the duration of the antifungal therapy and its type during preventive strategies, such as prophylaxis[9].

C. albicans susceptibility breakpoint is continuously updated by the Clinical and Laboratory Standards Institute (CLSI), which can be used as an indicator to manage systemic candidiasis [10]. Although $C$. albicans is the most common cause of Candida infections, the prevalence of non-albicans species is increasing [6]. The emergence of non-albicans Candida infections have become a global concern; however, as we described previously, change in its epidemiology after adherence to the AFS program was observed [11]. Similar positive effects could be expected by improving the susceptibility pattern of Candida spices after AFS implementation. Therefore, the aim of this study was to identify the antifungal susceptibility of Candida species amongst pediatric patients with Candida colonization and also to investigate the ASP intervention effects on antifungal susceptibility in pediatric patients with hematological malignancy.

The incidence of IFDs ranged from 7.7 to $12.5 / 1000$ admissions during 2015-18 in our center. Invasive candidiasis (IC) is the most common form of IFDs (47.2\%), and its annual incidence ranges $22.5-55.3 \%$.

\section{Methods And Materials}




\section{Settings}

The university hospital Amir is a tertiary oncology center in Shiraz, Iran, with more than 1.5 million inhabitants. The department of hematology/medical oncology has seven wards (100 active beds), an autologous transplant ward, and annually over 5000 pediatric patients are given admission.

\section{Population}

All children aged <18-year-old between 2017-18 with hematologic malignancy or solid organ tumors were included in this study. Samples were collected from oral/nasal secretions and urine/stool specimens. In children with severe thrombocytopenia or bleeding tendency, only urine and stool samples were collected. Weekly sampling was performed for patients with more than one-week of hospitalization. This cross-sectional study was approved by the Ethics Committee of Professor Alborzi Clinical Microbiology Research Center, Shiraz University of Medical Sciences. After explaining the study objectives, informed consent of children's parents or guardians was obtained.

\section{Mycological study}

Urine, nasal, oral, and stool samples were cultured on Sabouraud Dextrose Agar (Merck, Germany) medium. The cultured samples were transferred to the Mycology Laboratory of Professor Alborzi Clinical Microbiology Research Center for identification and susceptibility testing. The fungi isolates were cultured twice on Potato Dextrose Agar medium at $35^{\circ} \mathrm{C}$ for seven days to ensure the culture purity, and then the cultures were read at $530 \mathrm{~nm}$ by a spectrophotometer. C. parapsilosis ATCC-22019 and C. krusei-ATCC-6258 were used as standard quality control CLSI-recommended strains.

\section{Antifungal drugs}

The amphotericin B, caspofungin, voriconazole, fluconazole, posaconazole, and itraconazole powders (Sigma-Aldrich, Germany) were used to make serial dilutions. The broth microdilution method was applied to determine the susceptibility. CLSI M51-A, M59, and M60 followed in specifying the susceptibility pattern of isolates $[10,12,13]$. Inoculums suspension was prepared, using the spectrophotometric method at $530 \mathrm{~nm}$. The dilutions of 1:1000 was prepared in RPMI 1640 medium (Sigma-Aldrich, England), supplemented with 1.5\% agar and 2\% glucose at pH 7.0, using 0.165 M-morpholine propane sulfuric acid (MOPS). The final concentration was adjusted to $0.5 \mathrm{McFarland}$, with a volume of $100 \mu \mathrm{l}$ conidium dilution, were added to wells and incubated at $35^{\circ} \mathrm{C}$. Positive and negative wells (wells without drug and yeast, respectively) were considered for each species, and the minimum inhibitory concentration (MIC) was read visually after 24 and 48 hours. For amphotericin B, complete growth inhibition was considered as MIC value. Then, $\mathrm{MIC}_{50}$ and $\mathrm{MIC}_{90}$ values of the isolated species were calculated. The susceptibility pattern of each isolate was analyzed based on CLSI breakpoints [10].

\section{Antifungal Stewardship program principles in Amir medical oncology center}

AFS is a "strategic planning" that can be summarized in learning, training, and continuous practice to improve evidence-based skills in the management of IFDs in high-risk patients. By the sustained adherence to the AFS, indiscriminate use of antifungal agents, drug resistance, side effects and costs will be reduces. The AFS has been executed in our center since June 2015. Characteristics of AFS interventions summarized in Table 1. It should be noted that the diagnosis and treatment of the IFDs was significantly improved after the implementation of the AFS. Changing from empiric therapy to pre-emptive antifungal treatment strategies was accomplished by the application of nonculture-based methods, such as galactomannan (GM) antigen test and polymerase chain reaction (PCR). Therapeutic drug monitoring and antifungal susceptibility testing has become the standard of care for monitor serum voriconazole concentrations and targeted therapy since the early 2016. We used the original data from our recent investigation, which was conducted before the implementation of AFS during 2011-2012 in pediatric patients with malignancy (period-1; p1) [14]. This was done to compare the clinical impact of AFS interventions on antifungal susceptibility of different Candida spices with the present study (period-2; p2). 
Table 1. Main components of implemented AFS interventions in AMOC

Appropriate treatment of the suspected IFDs

Disposition to targeted therapy (by diagnostic driven approach) instead of empiric treatment

Adherence to current evidence-based guidelines in the treatment of the IFDs instead of individual decision making

Appropriate antifungal prescription

Appropriate antifungal selection

Appropriate duration

Appropriate administration route

Appropriate dosage

Limited use of azoles for prophylaxis of the IFDs (only for secondary prophylaxis in patients with a previous history of IMDs)

Regular epidemiologic surveillance to estimate fungal infection incidence and detection of any epidemiologic shift

Regular surveillance of the sensitivity pattern to antifungal drugs

- Appropriate use of new diagnostic modalities (implementation of routine GM test, twice/week during prolonged and profound neutropenic phase (ANC $<500$ cells $/ \mathrm{mm}^{3}$ )

Improving mycological diagnostic approach with judicious use of bronchoalveolar lavage and ultrasound/CT scan guided lung biopsy (or other organs as needed)

Time-sensitive automatic stop orders for specified antifungal prescriptions

Switching from intravenous to oral antifungal, when appropriate and confirmed by the infectious disease consultant

Full-time laboratory services (24-hour, seven days per week coverage) and strategies for reducing lab turnaround time (establishing a "hotline" for contributors to call about the lab test results)

Non-medical approach to prevent fungal infections

Applying modalities to reduce the nosocomial infections (for example diminished colonization by the appropriate use of an indwelling catheter)

Surveillance of the possible environmental roots of infection (for example surveillance of indoor spore load in the hospital's wards)

AFS: antifungal Stewardship; IFDs: invasive fungal diseases; IMDs: invasive mold diseases; GM: galactomannan; ANC: absolute neutrophil count; AMOC: Amir Medical Oncology Center,

\section{Statistical analysis}

Data were analyzed using IBM SPSS Statistics 21 software (IBM Corp. Released 2012. IBM SPSS Statistics for Windows, Version 21.0. Armonk, NY: IBM Corp.). All categorical variables are reported in percentages and numbers. P values were calculated, using the Chi-square test and Fisher's exact test. $\mathrm{P}$ values $<0.05$ were considered to be statistically significant. The Pearson correlation test was used to investigate the correlations between quantitative variables.

\section{Results}

From May 2017 to November 2018, 482 oral/nasal samples, and urine and stool specimens were collected from 136 pediatric patients with hematological malignancies or solid organ tumors. Most patients were male (53.3\%), and the mean age was 7.57 years (Median: 6.5 , Std. Deviation: \pm 4.85 , range from 4.8 months to 18 years). Eighty-two cases were detected having at least one Candida spp. colony, and amongst them 59 were $C$. albicans colonies.

Acute lymphoblastic leukemia (41/136, 30.1\%), acute myeloblastic leukemia (18/136, 13.2\%), and neuroblastoma (13/136, 9.5\%) were the most common underlying diseases, respectively. In total, $51.4 \%(70)$ were neutropenic (absolute neutrophil count $<1500$ cells $/ \mathrm{mm}^{3}$ ).

One hundred thirty-three strains of Candida spices were detected ( 2 species not identified), amongst which 102 strains were $C$. albicans followed by C. krusei (7), C. kefyr (7), C. parapsilosis (5), C. glabrata (4), C. tropicalis (3), and C. famata (3). Susceptibility of Candida species to different antifungal drugs are summarized in Table 2. 
Among the 102 C. albicans isolates collected during 2017-18 surveillance program, all were susceptible to the azole agents. The sensitivity rate of $C$. albicans to amphotericin B and caspofungin was $93.1 \%(95)$ and $97.1 \%(99)$, respectively.

Among the seven $C$. krusei isolates, $28.6 \%$ were resistant to caspofungin. All the $C$. krusei isolates were sensitive to amphotericin B, voriconazole. For itraconazole, $85.7 \%$ were sensitive, and $14.3 \%$ were susceptible dose-dependent.

All the five $C$. parapsilosis isolates were sensitive to amphotericin B, caspofungin. For itraconazole, $80 \%$ were sensitive, and $20 \%$ were susceptible dose-dependent. In total, $80 \%$ of $C$. parapsilosis were found to be susceptible to fluconazole.

Among all the tested strains of $C$. glabrata and $C$. tropicalis, none of them were resistant to any of the antifungal agents.

CLSI breakpoints are not available for $C$. Kefyr and $C$. famata. Susceptibility of different antifungals to $C$. kefyrand $C$. famata is provided in Table 3. 
Table 2

Susceptibility of 133 Candida spp. to antifungal drugs and distributions of MIC $(\mu \mathrm{g} / \mathrm{ml})$ by CLSI broth microdilution method ${ }^{\text {a }}$

\begin{tabular}{|c|c|c|c|c|c|c|c|c|c|c|c|c|}
\hline Organism & AF & Breakpoints & S & SDD & I & $\mathbf{R}$ & $\mathrm{ECV}^{*}$ & WT & N-WT & MIC50* & MIC90* & $\begin{array}{l}\text { MIC } \\
\text { Range* }^{*}\end{array}$ \\
\hline \multirow[t]{6}{*}{ C. albicans } & AmpB & $S \leq 1, R \geq 1$ & $93.1 \%$ & - & - & $6.9 \%$ & 4 & $96 \%$ & $4 \%$ & 0.250 & 0.50 & $0.032-8$ \\
\hline & CSF & $\begin{array}{l}S \leq 0.25, I \\
=0.5, R \geq 1\end{array}$ & $97.1 \%$ & - & $1.96 \%$ & $1 \%$ & 0.25 & $97 \%$ & $3 \%$ & 0.032 & 0.064 & $0.032-1$ \\
\hline & VCZ & $\begin{array}{l}S \leq 0.12, I \\
=0.25,-0.5 \\
R \geq 1\end{array}$ & $100 \%$ & - & - & - & 0.032 & $98 \%$ & $2 \%$ & 0.032 & 0.032 & $\begin{array}{l}0.032- \\
0.125\end{array}$ \\
\hline & FCZ & $\begin{array}{l}S \leq 2, \mathrm{SDD} \\
=4, \mathrm{R} \geq 8\end{array}$ & $100 \%$ & - & - & - & 0.25 & $98 \%$ & $2 \%$ & 0.032 & 0.125 & $0.032-4$ \\
\hline & PSZ & \multicolumn{5}{|c|}{ This drug doesn't have any breakpoint } & - & - & - & - & - & - \\
\hline & ITC & $\begin{array}{l}S \leq 0.12 \\
S D D=0.25 \\
-0.5 R \geq 1\end{array}$ & $100 \%$ & - & - & - & 0.064 & $98 \%$ & $2 \%$ & 0.032 & 0.032 & $\begin{array}{l}0.032- \\
0.064\end{array}$ \\
\hline \multirow[t]{6}{*}{ C. glabrata } & AmpB & $S \leq 1, R \geq 1$ & $100 \%$ & - & - & - & 0.25 & $75 \%$ & $25 \%$ & 0.250 & 0.5 & $\begin{array}{l}0.25- \\
0.5\end{array}$ \\
\hline & CSF & $\begin{array}{l}S \leq 0.12, I \\
=0.25, R \geq \\
0.5\end{array}$ & $75 \%$ & - & $25 \%$ & - & 0.125 & $75 \%$ & $25 \%$ & 0.125 & 0.25 & $\begin{array}{l}0.064- \\
0.25\end{array}$ \\
\hline & VCZ & \multicolumn{5}{|c|}{$\mathrm{ECV}=0.5, \mathrm{WT}: \mathrm{MIC} \leq \mathrm{ECV} \&$ Non-WT: $\mathrm{MIC}>\mathrm{ECV}$} & 0.032 & $100 \%$ & - & 0.032 & 0.032 & 0.032 \\
\hline & FCZ & $\begin{array}{l}\mathrm{SDD} \leq 32 \\
\mathrm{R} \geq 64\end{array}$ & - & $100 \%$ & - & - & 0.25 & $75 \%$ & $25 \%$ & 0.25 & 1 & $0.125-1$ \\
\hline & PSZ & \multicolumn{5}{|c|}{ This drug doesn't have any breakpoint } & - & - & - & - & - & - \\
\hline & ITC & $\begin{array}{l}S \leq 0.12 \\
S D D=0.25 \\
-0.5 R \geq 1\end{array}$ & $100 \%$ & - & - & - & 0.064 & $75 \%$ & $25 \%$ & 0.064 & 0.125 & $\begin{array}{l}0.064- \\
0.125\end{array}$ \\
\hline \multirow[t]{6}{*}{ C. krusei } & AmpB & $S \leq 1, R \geq 1$ & $100 \%$ & - & - & - & 0.5 & $85.7 \%$ & $28.6 \%$ & 0.5 & 1 & $0.25-1$ \\
\hline & CSF & $\begin{array}{l}S \leq 0.25, I \\
=0.5, R \geq 1\end{array}$ & $14.3 \%$ & - & $57.1 \%$ & $28.6 \%$ & 0.5 & $71.4 \%$ & $14.3 \%$ & 0.5 & 1 & $0.25-1$ \\
\hline & VCZ & $\begin{array}{l}\mathrm{S} \leq 0.5, \mathrm{I}= \\
1, \mathrm{R} \geq 2\end{array}$ & $100 \%$ & - & - & - & 0.125 & $85.7 \%$ & $28.6 \%$ & 0.125 & 0.25 & $\begin{array}{l}0.064- \\
0.25\end{array}$ \\
\hline & $\mathrm{FCZ}$ & \multicolumn{5}{|c|}{$\begin{array}{l}\text { C. krusei is considered resistant to FCZ, } \\
\text { irrespective of the MIC }\end{array}$} & - & - & - & - & - & - \\
\hline & PSZ & \multicolumn{5}{|c|}{ This drug doesn't have any breakpoint } & - & - & - & - & - & - \\
\hline & ITC & $\begin{array}{l}S \leq 0.12 \\
S D D=0.25 \\
-0.5 R \geq 1\end{array}$ & $85.7 \%$ & $14.3 \%$ & - & - & 0.125 & $85.7 \%$ & $28.6 \%$ & 0.125 & 0.25 & $\begin{array}{l}0.125- \\
0.25\end{array}$ \\
\hline \multirow[t]{4}{*}{ C. tropicalis } & AmpB & $S \leq 1, R \geq 1$ & $100 \%$ & - & - & - & 0.25 & $66.7 \%$ & $33.3 \%$ & 0.25 & 0.5 & $0.25-.5$ \\
\hline & CSF & $S \leq 2$ & $100 \%$ & - & - & - & 0.064 & $66.7 \%$ & $33.3 \%$ & 0.064 & 1 & $.032-1$ \\
\hline & VCZ & $\begin{array}{l}S \leq 0.12, I \\
=0.25,-0.5 \\
R \geq 1\end{array}$ & $100 \%$ & - & - & - & 0.032 & $100 \%$ & - & 0.032 & 0.032 & 0.032 \\
\hline & FCZ & $\begin{array}{l}S \leq 8, R \geq \\
64\end{array}$ & $100 \%$ & - & - & - & 0.032 & $66.7 \%$ & $33.3 \%$ & 0.032 & 0.125 & $\begin{array}{l}0.032- \\
0.25\end{array}$ \\
\hline
\end{tabular}

* $(\mu \mathrm{g} / \mathrm{ml})$. a. Based on recommended CLSI 24-hour minimum inhibitory concentration limits [10].

AmpB: Amphotericin B, CSF: Caspofungin, VCZ: Voriconazole, FCZ: Fluconazole, PSZ: Posaconazole, ITC: Itraconazole, AF: antifungal, SDD: susceptible dose-dependent, S: sensitive, I: intermediate, R: resistant, ECV: Epidemiological Cutoff Value; ECVs capture $\geq 97.5 \%$ of the statistically modelled population [12], WT: Wild-type, NWT: non-wild-type, MIC50: Minimum Inhibitory Concentration required to inhibit the growth of $50 \%$ of organisms. MIC90: Minimum Inhibitory Concentration required to inhibit the growth of $90 \%$ of fungal spices. 


\begin{tabular}{|c|c|c|c|c|c|c|c|c|c|c|c|c|}
\hline Organism & AF & Breakpoints & $s$ & SDD & 1 & $\mathbf{R}$ & $\mathrm{ECV}^{*}$ & WT & N-WT & MIC50* & MIC90* & $\begin{array}{l}\text { MIC } \\
\text { Range* }\end{array}$ \\
\hline & PSZ & \multicolumn{5}{|c|}{ This drug doesn't have any breakpoint } & - & - & - & - & - & - \\
\hline & ITC & $\begin{array}{l}S \leq 0.12 \\
S D D=0.25 \\
-0.5 R \geq 1\end{array}$ & $100 \%$ & - & - & - & 0.032 & $100 \%$ & - & 0.032 & 0.032 & 0.032 \\
\hline \multirow{6}{*}{$\begin{array}{l}\text { C. } \\
\text { parapsilosis }\end{array}$} & AmpB & $S \leq 1, R \geq 1$ & $100 \%$ & - & - & - & 0.25 & $80 \%$ & $20 \%$ & 0.25 & 0.5 & $.032-.5$ \\
\hline & CSF & $\begin{array}{l}S \leq 2, I=4 \\
R \geq 8\end{array}$ & $100 \%$ & - & - & - & 0.5 & $80 \%$ & $20 \%$ & 0.064 & 0.125 & $\begin{array}{l}.032- \\
0.125\end{array}$ \\
\hline & VCZ & $\begin{array}{l}S \leq 0.12, I \\
=0.25,-0.5 \\
R \geq 1\end{array}$ & $80 \%$ & - & $20 \%$ & - & 0.032 & $80 \%$ & $20 \%$ & 0.032 & 0.5 & $.032-.5$ \\
\hline & $\mathrm{FCZ}$ & $\begin{array}{l}S \leq 2, S D D \\
=4, R \geq 8\end{array}$ & $80 \%$ & - & - & $20 \%$ & 0.064 & $80 \%$ & $20 \%$ & 0.064 & 16 & $\begin{array}{l}.032- \\
16\end{array}$ \\
\hline & PSZ & \multicolumn{5}{|c|}{ This drug doesn't have any breakpoint } & - & - & - & - & - & - \\
\hline & ITC & $\begin{array}{l}S \leq 0.12 \\
S D D=0.25 \\
-0.5 R \geq 1\end{array}$ & $80 \%$ & $20 \%$ & - & - & 0.032 & $80 \%$ & $20 \%$ & 0.032 & 0.25 & $.032-.25$ \\
\hline \multicolumn{13}{|c|}{ * $(\mu \mathrm{g} / \mathrm{ml})$. a. Based on recommended CLSI 24-hour minimum inhibitory concentration limits [10]. } \\
\hline \multicolumn{13}{|c|}{$\begin{array}{l}\text { AmpB: Amphotericin B, CSF: Caspofungin, VCZ: Voriconazole, FCZ: Fluconazole, PSZ: Posaconazole, ITC: Itraconazole, AF: antifungal, } \\
\text { SDD: susceptible dose-dependent, S: sensitive, I: intermediate, R: resistant, ECV: Epidemiological Cutoff Value; ECVs capture } \geq 97.5 \% \text { of } \\
\text { the statistically modelled population [12], WT: Wild-type, NWT: non-wild-type, MIC50: Minimum Inhibitory Concentration required to } \\
\text { inhibit the growth of } 50 \% \text { of organisms. MIC90: Minimum Inhibitory Concentration required to inhibit the growth of } 90 \% \text { of fungal spices. }\end{array}$} \\
\hline
\end{tabular}

Table 3

Susceptibilities of different antifungals to C. kefyr and C. famata

\begin{tabular}{|lllll|}
\hline \multirow{2}{*}{$\begin{array}{l}\text { Species } \\
\text { (no. tested) }\end{array}$} & Antifungal agent & & $\mathbf{M I C}(\boldsymbol{\mu g} / \mathrm{ml})$ & \\
\cline { 3 - 5 } C. kefyr $(7)$ & Fluconazole & $0.032-0.25$ & 0.064 & 0.125 \\
\hline & Voriconazole & 0.032 & 0.032 & 0.032 \\
\hline & Itraconazole & 0.032 & 0.032 & 0.032 \\
\hline & Caspofungin & $0.032-1$ & 0.064 & 0.125 \\
\hline & Amphotericin B & $0.064-2$ & 0.25 & 0.5 \\
\hline C. famata (3) & Fluconazole & $0.032-0.25$ & 0.032 & 0.032 \\
& Voriconazole & 0.032 & 0.032 & 0.032 \\
\hline & Itraconazole & $0.032-0.25$ & 0.032 & 0.032 \\
\hline & Caspofungin & $0.032-0.125$ & 0.032 & 0.032 \\
\hline & Amphotericin B & $0.25-0.5$ & 0.25 & 0.25 \\
\hline
\end{tabular}

The epidemiologic cutoff value (ECV) for in vitro susceptibility testing of Candida spp. was calculated based on "CLSI epidemiological cutoff values for antifungal susceptibility testing. 2nd ed. CLSI supplement M59" [12].

CLSI does not provide posaconazole minimal inhibitory concentration breakpoint for $C$. albicans. Posaconazole 24-hour and 48-hour MIC statistics were determined for 106 C. albicans isolates. Accordingly, mean 24-hour and 48-hour MIC were 0.0361 and 0.0394 , respectively (Table 4, Fig. 1). 
Table 4

Posaconazole 24-hour and 48-hour MIC statistics for 102 isolates of C. albicans

\begin{tabular}{|lll|}
\hline & 24-hour MIC & 48-hour MIC \\
\hline Mean & 0.0361 & 0.0394 \\
\hline Median & 0.0320 & 0.0320 \\
\hline Mode & 0.03 & 0.03 \\
\hline Std. Deviation & 0.02470 & 0.04727 \\
\hline Variance & 0.001 & 0.002 \\
\hline Range & 0.22 & 0.47 \\
\hline Minimum & 0.03 & 0.03 \\
\hline Maximum & 0.25 & 0.50 \\
\hline
\end{tabular}

\section{Discussion}

Amongst the 136 studied cases, 59.9\% (82) were colonized with at least one Candida species. Most of them were colonized with C. albicans 59 (72\%). C. krusei, C. kefyr, C. glaberata, C. parapsilosis, C. tropicalis, and C. famata were the least common Candida species. Detail information regarding the colonization pattern of the studied cases can be found in our recently published paper [11].

According to the results, all C. krusei, C. glaberata, C. parapsilosis, and C. tropicalis isolates were susceptible to amphotericin B (the most active agent for the treatment of non-albicans Candida species). Caspofungin exhibited high resistance against $C$. krusei and $C$. glaberata isolates ( $28.6 \%$ and $25 \%$, respectively). Accordingly, amphotericin B can be considered as a more active agent than caspofungin for the treatment of non-albicans Candida species, especially, C. krusei and C. glaberata.

\section{Frequency of azoles, caspofungin and amphotericin B resistance amongst uncommon Candida spices}

There are limited reports on azole MIC range for $C$. kefyr. However, based on a large epidemiological study in our region [8], the available MIC range in colonizing isolates were 0.064-2 (MIC 90\%: $0.5 \mu \mathrm{g} / \mathrm{ml}$ ); 0.032-0.25 (MIC 90\%: $0.125 \mu \mathrm{g} / \mathrm{ml}$ ); 0.032-0.125 (MIC 90\%: $0.032 \mu \mathrm{g} / \mathrm{ml}$ ) for fluconazole, itraconazole and voriconazole, respectively. The caspofungin MIC range was reported between 0.032-2 (MIC 50\%: $0.15 \mu \mathrm{g} / \mathrm{ml}$ and MIC $90 \%: 0.03 \mu \mathrm{g} / \mathrm{ml}$ ) [8, 15]. C. kefyris generally considered susceptible to amphotericin B $(\mathrm{MIC}<1 \mu \mathrm{g} / \mathrm{ml})$; however, wide range of MICs have been reported worldwide (0.5-64) [16-18]. C. kefyr is usually considered susceptible to posaconazole (MIC $90 \%$ : 0.032 to $0.12 \mu \mathrm{g} / \mathrm{ml})[8,17]$.

The reported fluconazole MIC range for $C$. famata is $0.25-16$ (MIC $50 \%: 2-4 \mu \mathrm{g} / \mathrm{ml}$ and MIC $90 \%: 16 \mu \mathrm{g} / \mathrm{ml}$ ) [19]; for itraconazole is $0.06-2$ (MIC 50\%: $0.25 \mu \mathrm{g} / \mathrm{ml}$ and MIC 90\%: $0.5 \mu \mathrm{g} / \mathrm{ml}$ ) [20]; and for voriconazole is 0.032-1 (MIC 90\%: $0.125 \mu \mathrm{g} / \mathrm{ml}$ ) [8]. The caspofungin MIC ragne is $0.032-0.25$ (MIC $50 \%: 0.5 \mu \mathrm{g} / \mathrm{ml}$ and MIC $90 \%: 1 \mu \mathrm{g} / \mathrm{ml}$ ) [8, 15]. C. famata is generally considered susceptible to amphotericin B $(\mathrm{MIC}<1 \mu \mathrm{g} / \mathrm{ml})[8,21]$. While higher posaconazole MICs were reported for $C$. famata $(0.015-1 ; \mathrm{MIC} 50 \%: 0.25 \mu \mathrm{g} / \mathrm{ml}$, and MIC $90 \%: 1 \mu \mathrm{g} / \mathrm{ml})$ [22], we found lower MIC range in our study $(0.032-0.125$, MIC $50 \%: 0.032 \mu \mathrm{g} / \mathrm{ml})$. It should be noted that MIC ranges are different between colonized and infected isolates [8].

Accordingly, in this study the colonized isolates of $C$. Kefyr and $C$. famata were susceptible to the tested antifungal agents.

Therapeutic options for fungal infections are limited even before the global rise of antifungal resistance [23, 24]; hence, judicious prescription of available choices, especially non-azole antifungals, should be considered in high-risk settings, such as oncology centers. Our results confirmed that caspofungin and amphotericin B are more active antifungal agents compare to azoles in some isolates, such as $C$. parapsilosis.

The emergence of azole-resistant $C$. glabrata is also of significant concern in the setting that use fluconazole prophylaxis [9].

\section{Clinical impact of antifungal stewardship interventions on antifungal susceptibility of Candida species during the two study periods}

Epidemiological changes in the Candida colonization pattern was described in our previous report. Briefly, we found a significant reduction in non-albicans species colonization after the implementation of AFS. During period 1 ( $p 1), 46.5 \%(88)$ of the studied cases $(n=188)$ were 
colonized, while in the 2 nd period, the colonization rate reached $59.9 \%$ ( $P$ value $=0.0 .017$ ) [11]. In total, $25.3 \%(23)$ of the cases were receiving antifungal prophylaxis during the 2 nd period, mainly with the liposomal formulation of amphotericin $B$, while $54 \%$ were on antifungal prophylaxis during $\mathrm{p} 1$, mostly with fluconazole or itraconazole [Difference $21.2 \%, 95 \% \mathrm{Cl}$ : $9.16-31.77 \%, \mathrm{P}=0.0007$ ]. This success was achieved by controlling and restricting antifungal usage during $\mathrm{p} 2$.

In a study by Hadadi et al. during 2011-12 C. albicans was the most common species followed by C. krusei, C. glabrata, C. tropicalis, $C$. famata, C. parapsilosis, C. dubliniensis, and C. kefyrduring the p1. C. glabrata was the most resistant isolated Candida species, showing $70 \%$ resistant to fluconazole and $50 \%$ to itraconazole, $7.5 \%$ to amphotericin $\mathrm{B}$, and $14 \%$ to ketoconazole [25].

\section{Frequency of azole and caspofungin resistance $\mathrm{C}$. albicans}

Amongst the 117 tested isolates of $C$. albicans, $52.5 \%$ (53) of the isolates were found to be azole-resistant during p1, while only $1.5 \%$ (2) were resistant during p2 (P value $<0.001)$. No fluconazole-resistant $(\mathrm{MIC} \geq 8 \mu \mathrm{g} / \mathrm{ml})$ C. albicans was detected during p2 (P value $<0.001)$. Multidrug-resistant strains, including azole, caspofungin and amphotericin B resistant isolates were not found within the two study periods (Table 4 and Fig. 2).

\section{Frequency of amphotericin B resistance $\mathrm{C}$. albicans}

Despite the significant reduction in fluconazole and caspofungin-resistant, during p2, some increase in the incidence of amphotericin Bresistant $C$. albicans was detected during p2 (Table 5). A small but significant increase in amphotericin B-resistant $C$. albicans strains can be partially explained by changing antifungal preventive strategy to liposomal amphotericin B, since 2015. However, the frequency of amphotericin B-resistant $C$. albicans was not affected by liposomal amphotericin B prophylaxis between the two periods $(p=0.619)$.

Table 5

The sensitivity of isolated $C$. albicans against fluconazole, caspofungin, and amphotericin $\mathrm{B}$, during 2011-12 (period 1) and 2017-18 (period 2), No. (\%) of isolates

\begin{tabular}{|lllll|}
\hline Antifungal agent & Sensitivity & Period 1 & Period 2* & p-value \\
\hline Fluconazole & Sensitive & $53(67.1)$ & $102(102)$ & $<0.001$ ** \\
\cline { 2 - 5 } & Resistant & $26(32.9)$ & 0 & \\
\hline Caspofungin & Sensitive & $54(83.1)$ & $99(97.1)$ & $<0.001$ ** \\
\cline { 2 - 5 } & Resistant & $11(16.9)$ & $1(1)$ & $<0.001$ ** \\
\hline Amphotericin B & Sensitive & $83(100)$ & $95(93.1)$ & \\
\cline { 2 - 5 } & Resistant & 0 & $7(6.9)$ & \\
\hline * No fluconazole-resistant isolates of C. albicans was found during period 2 (2017-2018)
\end{tabular}

Frequency of azoles, caspofungin, and amphotericin B resistance amongst the non -albicans colonized species

We also analyzed the rate of fluconazole, voriconazole, itraconazole, caspofungin and amphotericin B resistance amongst the non-albicans colonized species between the two study periods. Significant decrease in fluconazole, itraconazole and caspofungin resistance was found among the $C$. glaberata strains during the second study period compared with 2011-12. Also, a statistically significant reduction in amphotericin B resistance was found during $\mathrm{p} 2$ in $\mathrm{C}$. krusei isolates (Table 6).

\section{Clinical impact of Candida colonization in hospitalized patients with hematological malignancies}

Most of Candida bloodstream infections, including central line-associated candidemia originate from endogenous host flora [26-28]. The clinical impact of Candida colonization on the short-term mortality rate of patients with hematological malignancies was reported in a previous study [29]. On the other hand, short-term survival was affected in patients with non-albicans species, including C. glabrata, C. kefyr, and $C$. krusei, compare with $C$. albicans $[29,30]$. Azole prophylaxis has a critical role in the development of either unsusceptible strains or even in the selection of yeast with intrinsic azole-resistant, such as $C$. krusei $[6,9,31,32]$.

As discussed earlier, during p1, more than $35 \%$ of cases were colonized with non-albicans species, mostly C. glabrata and C. krusei. However, after the implementation of the AFS, non-albicans colonization decreased to less than $20 \%$, mostly C. krusei and C. Parapsilosis, with a significant decrease in C. glabrata colonization [11]. C. glabrata is considered as the second most common gastrointestinal yeast 
flora, after $C$. albicans [33]. In our opinion, the shift toward more $C$. albicans colonization is the result of sustained adherence to the AFS and restricted azole prescription (Table 1).

Comparison of the mean minimum inhibitory concentration (MIC) value of the six antifungal drugs between different Candida spp. during the two study periods

In addition to the susceptibility results, we also compared the mean MIC value of each antifungal drug for $C$. albicans, $C$. glabrata, $C$. krusei, C. parapsilosis, C. tropicalis, C. famata, and C. kefyrduring two study periods (Fig. 4). As shown in Fig. 4, a significant reduction in mean FCZ-MIC was found for $C$. albicans, C. glabrata, and C. tropicalis in p2 compare to $\mathrm{p} 1$.

There are lots of data with respect to the positive effect of stewardship program on bacterial resistance [34-37]; however, due to its multifactorial development, antifungal resistance is more challenging to measure. Even in colonized patients, susceptibility patterns might change over time, especially in immunocompromised hosts [8]. Although the AFS has known short-term effects (e.g., reduction in antifungal consumption, costs and outcomes) on the management of IFDs and patient safety [38-41], its long-term effects has been described on resistance patterns [42]. Based on stewardship program metrics, change in resistance patterns, and pathogen-specific resistance is the most difficult target to reach [34]. To the best of our knowledge, there is a shortage of reports on the improvement of antifungal susceptibility of Candida species overtimes after the implementation of AFS. Hence, the results of our study highlight the importance of strict adherence to the stewardship programs amongst the cancer patients.

\section{Limitations}

This study was limited by the small number of samples. Additionally, further studies using next generation sequencing are needed to detect AFS effects on antifungal resistance genes in Candida spices.

\section{Conclusion}

C. albicans is the most prevalent colony amongst the pediatric patients with malignancy, and azoles remain the most effective choice when they are used wisely. Improving Candida species antifungal susceptibility after the implementation of AFS is promising. Knowledge of etiologic agents, along with the regular identification of antifungal susceptibility patterns is necessary in oncology settings.

\section{Declarations}

\section{Ethics and consent to participate}

The study was approved by medical ethics committee of Professor Alborzi clinical microbiology research center. All individuals (or their parents) in the study population were informed about the current study, with written consents obtained before enrolment in the present study.

\section{Consent for publication}

Not applicable

\section{Availability of data and material}

The datasets used and/or analyzed during the current study are available from the corresponding author on reasonable request.

\section{Competing interests}

The authors do not have any financial or other relationships which could be regarded as a conflict of interest.

\section{Funding}

Not applicable

\section{Author contributions:}


Study concept and design: AA and HSS; Acquisition of data: AA, JH, HSS and GF; Mycological analysis: BP, JH and GF; Statistical Analysis: AA, HS, and NS, Analysis and interpretation of data: AA and HSS; Drafting of the manuscript: AA, Critical revision of the manuscript for important intellectual content: AA and BP; Study supervision: AA and BP. All individuals listed as (co)-authors have met the authorship criteria, and nobody who qualifies for authorship has been omitted from the list. The final manuscript was corrected and approved by all authors.

\section{Acknowledgment}

The authors wish to thank Mr. H. Argasi at the Research Consultation Center (RCC) of Shiraz University of Medical Sciences for his invaluable assistance in editing this manuscript.

\section{References}

1. Richardson M, Lass C, Flörl. Changing epidemiology of systemic fungal infections. Clin Microbiol Infect. 2008;14:5-24.

2. Brown GD, et al., Hidden killers: human fungal infections. Science translational medicine, 2012. 4(165): p. 165rv13-165rv13.

3. Sweileh WM, et al. Bibliometric analysis of literature on antifungal triazole resistance: 1980-2015. Germs. 2017;7(1):19.

4. Zand F, et al. Invasive fungal infections in critically-ill patients: A literature review and position statement from the IFI-clinical forum, Shiraz, Iran. Biosci Biotechnol Res Communicat. 2016;9(3):371-81.

5. Badiee P, Alborzi A. Susceptibility of clinical Candida species isolates to antifungal agents by E-test, Southern Iran: A five year study. Iranian journal of microbiology. 2011;3(4):183.

6. Jensen RH. Resistance in human pathogenic yeasts and filamentous fungi: prevalence, underlying molecular mechanisms and link to the use of antifungals in humans and the environment. Dan Med J. 2016;63:1-34.

7. Gonçalves SS, et al. Epidemiology and molecular mechanisms of antifungal resistance in Candida and Aspergillus. Mycoses. 2016;59(4):198-219.

8. Badiee P, et al. Antifungal susceptibility testing of Candida species isolated from the immunocompromised patients admitted to ten university hospitals in Iran: comparison of colonizing and infecting isolates. BMC Infect Dis. 2017;17(1):727.

9. Kullberg BJ, Arendrup MC. Invasive candidiasis. N Engl J Med. 2015;373(15):1445-56.

10. Clinical and Laboratory Standards Institute. Performance Standards for Antifungal Susceptibility' Testing of Yeasts. CLSI supplement M60. 2017: USA. 950 West Valley Road. Suite 2500. Wayne. Pennsylvania 19087.

11. Hamzavi SS, et al., Changing face of Candida colonization pattern in pediatric patients with hematological malignancy during repeated hospitalizations, results of a prospective observational study (2016-2017) in shiraz, Iran. BMC infectious diseases, 2019. 19 (1): p. 759.

12. Clinical and Laboratory Standards Institute, Epidemiological Cutoff Values for Antifungal Susceptibility Testing. 2nd ed. CLSI supplement M59. 2018: 950 West Valley Road, Suite 2500, Wayne, Pennsylvania 19087 USA, 2018.

13. Clinical and Laboratory Standards Institute, Method for Antifungal Disk Diffusion Susceptibility Testing of Nondermatophyte Filamentous Fungi; Approved Guideline. C'LSI document M51-A. 2010: 940 West Valley Road. Suite 1400. Wayne. Pennsylvania 19087 - 1898 USA.

14. Badiee P, et al. Susceptibility of Candida species isolated from immunocompromised patients to antifungal agents. East Mediterr Health J. 2011;17(5):425-30.

15. Russell E, Lewis NDB, Dimitrios P, Kontoyiannis, Caspofungin, in Kucers' The Use of Antibiotics Seventh Edition: A Clinical Review of Antibacterial, Antifungal and Antiviral Drugs, M. Lindsay Grayson, et al., Editors. 2018, CRC Press. p. 2661.

16. Neil RH, Stone TB, Amphotericin B Deoxycholate, in Kucers' The Use of Antibiotics Seventh Edition: A Clinical Review of Antibacterial, Antifungal and Antiviral Drugs, M. Lindsay Grayson, et al., Editors. 2018, CRC Press. p. 2572.

17. Diekema $D$, et al. In vitro activity of seven systemically active antifungal agents against a large global collection of rare Candida species as determined by CLSI broth microdilution methods. J Clin Microbiol. 2009;47(10):3170-7.

18. Barchiesi F, et al. In-vitro activity of five antifungal agents against uncommon clinical isolates of Candida spp. J Antimicrob Chemother. 1999;43(2):295-9.

19. Sharon CA, Chen TCS, Fluconazole, in Kucers' The Use of Antibiotics Seventh Edition: A Clinical Review of Antibacterial, Antifungal and Antiviral Drugs, M. Lindsay Grayson, et al., Editors. 2018, CRC Press. p. 2757.

20. Jeniel E, Nett DRA, Itraconazole, in Kucers' The Use of Antibiotics Seventh Edition: A Clinical Review of Antibacterial, Antifungal and Antiviral Drugs, M. Lindsay Grayson, et al., Editors. 2018, CRC Press. p. 2787.

Page $11 / 15$ 
21. Neil RH, Stone TB, Amphotericin B Deoxycholate, in Kucers' The Use of Antibiotics Seventh Edition: A Clinical Review of Antibacterial, Antifungal and Antiviral Drugs, M. Lindsay Grayson, et al., Editors. 2018, CRC Press. p. 2570.

22. Fedja Farowski OAC, Posaconazole, in Kucers' The Use of Antibiotics Seventh Edition: A Clinical Review of Antibacterial, Antifungal and Antiviral Drugs, M. Lindsay Grayson, et al., Editors. 2018, CRC Press. p. 2845.

23. Centers for Disease Control and Prevention, Antibiotic Resistance Threats in the United States. 2019, Atlanta, GA: U.S.: Department of Health and Human Services, CDC; 2019.

24. Organization WH, Antimicrobial resistance: global report on surveillance. 2014: World Health Organization.

25. Haddadi P, et al., Yeast colonization and drug susceptibility pattern in the pediatric patients with neutropenia. Jundishapur journal of microbiology, 2014. 7(9).

26. Hovi L, et al. Invasive fungal infections in pediatric bone marrow transplant recipients: single center experience of 10 years. Bone marrow transplantation. 2000;26(9):999-1004.

27. Delaloye J, Calandra T. Invasive candidiasis as a cause of sepsis in the critically ill patient. Virulence. 2014;5(1):161-9.

28. Sendid B, Lacroix C, Bougnoux M-E. Is Candida kefyr an emerging pathogen in patients with oncohematological diseases? Clinical infectious diseases, 2006. 43(5): p. 666-667.

29. Safdar A, Armstrong D. Prospective evaluation of Candida species colonization in hospitalized cancer patients: impact on short-term survival in recipients of marrow transplantation and patients with hematological malignancies. Bone marrow transplantation.

2002;30(12):931-5.

30. Pfaller MA, et al., Epidemiology and outcomes of invasive candidiasis due to non-albicans species of Candida in 2,496 patients: data from the Prospective Antifungal Therapy (PATH) registry 2004-2008. PloS one, 2014. 9(7).

31. Safdar A, et al. Candida glabrata and Candida krusei fungemia after high-risk allogeneic marrow transplantation: no adverse effect of low-dose fluconazole prophylaxis on incidence and outcome. Bone marrow transplantation. 2001;28(9):873-8.

32. Sendid B, et al. Candidaemia and antifungal therapy in a French University Hospital: rough trends over a decade and possible links. BMC Infect Dis. 2006;6(1):80.

33. Bougnoux $M-E$, et al. Multilocus sequence typing reveals intrafamilial transmission and microevolutions of Candida albicans isolates from the human digestive tract. J Clin Microbiol. 2006;44(5):1810-20.

34. BEGANOVIC M, LAPLANTE KL, Communicating with Facility Leadership; Metrics for Successful Antimicrobial Stewardship Programs (ASP) in Acute Care and Long-Term Care Facilities. Rhode Island medical journal (2013), 2018. 101(5): p. 45-49.

35. Dancer S, et al. Approaching zero: temporal effects of a restrictive antibiotic policy on hospital-acquired Clostridium difficile, extendedspectrum $\beta$-lactamase-producing coliforms and meticillin-resistant Staphylococcus aureus. Int J Antimicrob Agents. 2013;41(2):13742.

36. Cook PP, Gooch M. Long-term effects of an antimicrobial stewardship programme at a tertiary-care teaching hospital. Int J Antimicrob Agents. 2015;45(3):262-7.

37. Hwang H, Kim B. Impact of an infectious diseases specialist-led antimicrobial stewardship programmes on antibiotic use and antimicrobial resistance in a large Korean hospital. Scientific reports. 2018;8(1):1-10.

38. Rac H, et al., impact of an antifungal stewardship intervention on optimization of candidemia management. Therapeutic advances in infectious disease, 2018. 5(1): p. 3-10.

39. Valerio M, et al., Antifungal stewardship in a tertiary-care institution: a bedside intervention. Clin Microbiol Infect, 2015. 21(5): 492. e1492. e9.

40. Rautemaa-Richardson $\mathrm{R}$, et al. impact of a diagnostics-driven antifungal stewardship programme in a UK tertiary referral teaching hospital. J Antimicrob Chemother. 2018;73(12):3488-95.

41. Hart E, et al. A systematic review of the impact of antifungal stewardship interventions in the United States. Ann Clin Microbiol Antimicrob. 2019;18(1):24.

42. Apisarnthanarak A, Yatrasert A, Mundy LM. Impact of education and an antifungal stewardship program for candidiasis at a Thai tertiary care center. Infection Control Hospital Epidemiology. 2010;31(7):722-7.

\section{Figures}



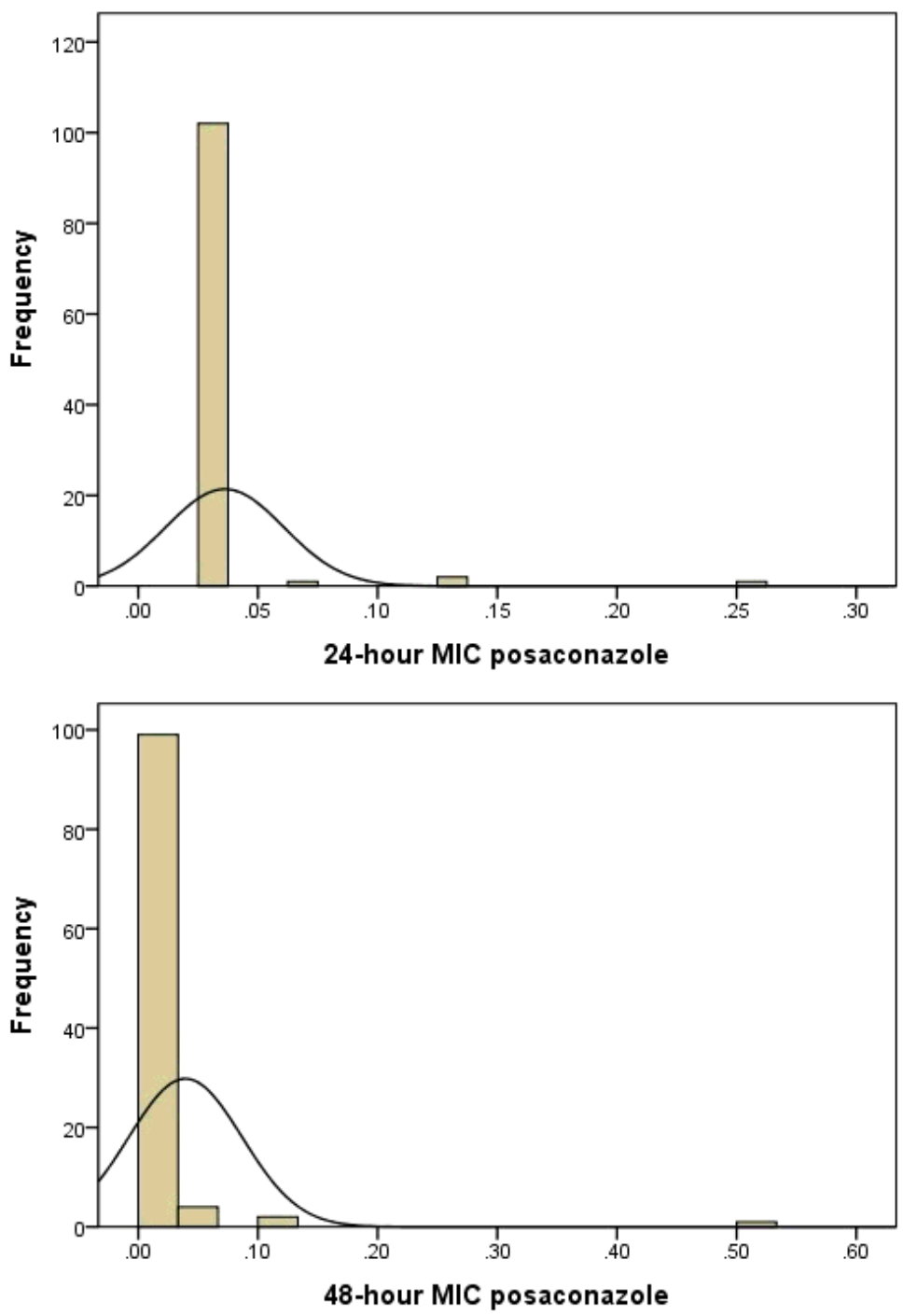

Figure 1

24-hour and 48-hour MIC distribution with a histogram of the isolated C. albicans 

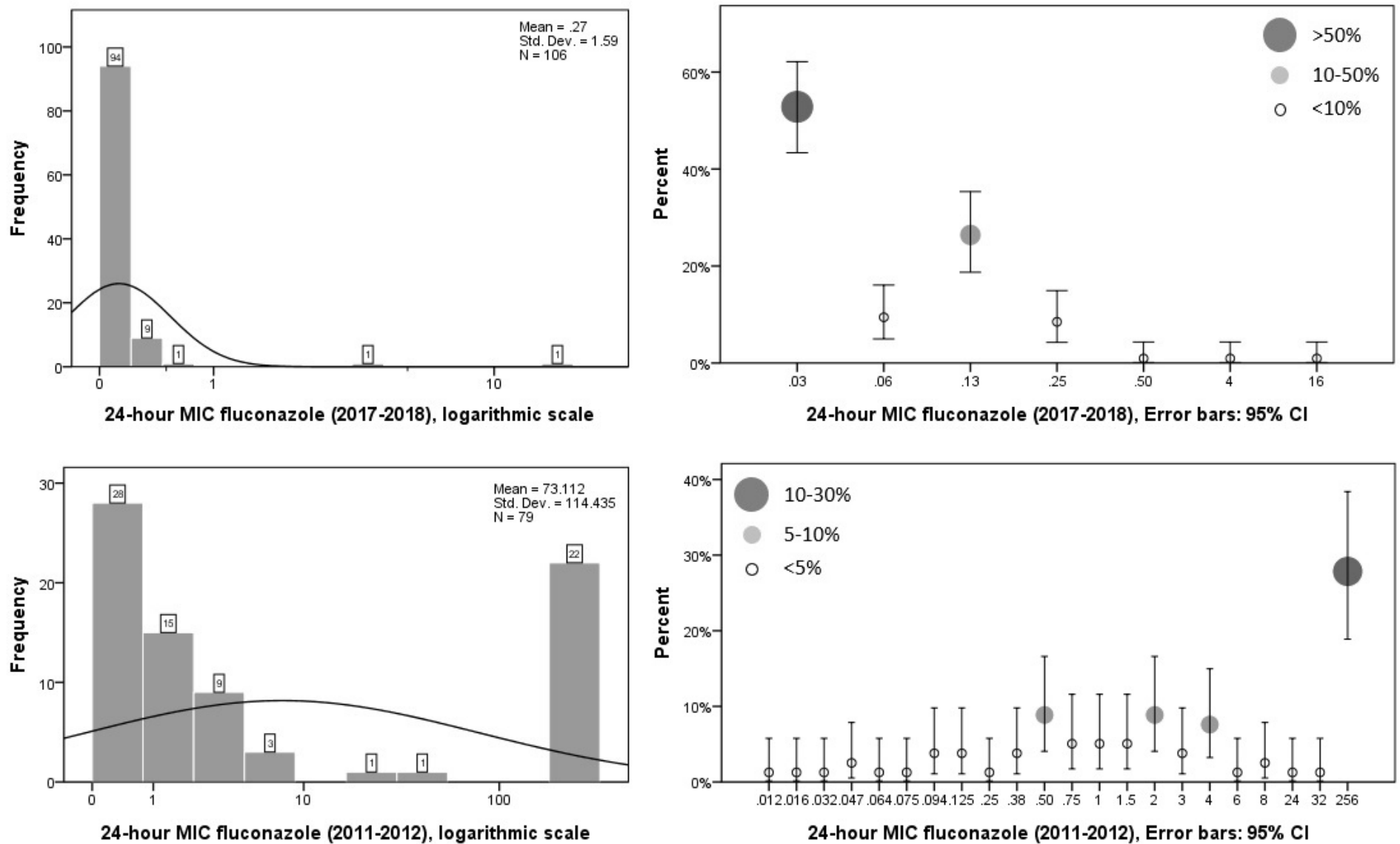

\section{Figure 2}

24-hour MIC fluconazole of 117 (2011-2012), and 106 (2017-2018) strains of C. albicans. Left, chart bar. Logarithmic scales of 24-hour MIC fluconazole, each bar is labelled with the number of isolates. MIC distribution histogram also is provided for better comparison between the two periods. Right, Error bar. Frequency of MIC results is presented in error bars with $95 \% \mathrm{Cl}$. Each error bar is labelled by circles that are representative of MIC frequency.

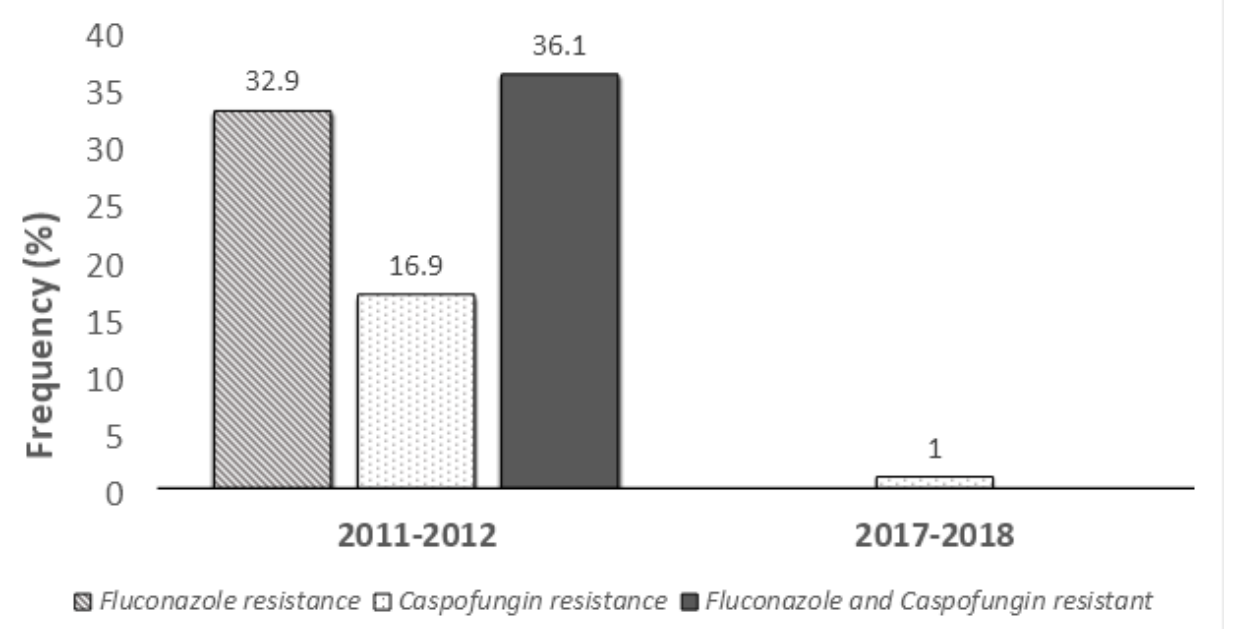

\section{Figure 3}

Frequency of fluconazole-resistant, caspofungin-resistant and fluconazole/caspofungin-resistant strains of $\mathrm{C}$. albicans during the two study periods 

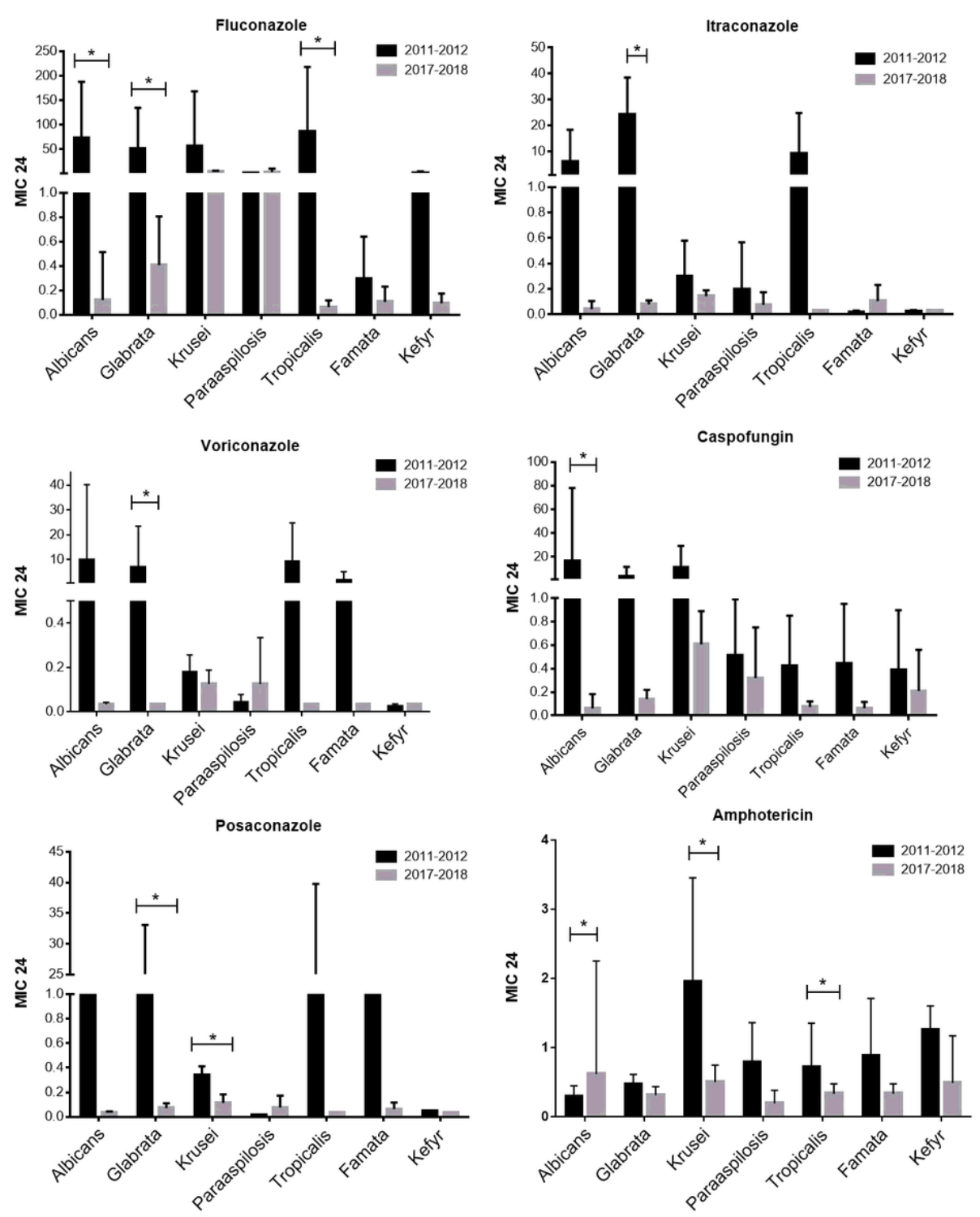

Figure 4

The mean MIC value (24-hour) of C. albicans, C. glabrata, C. krusei, C. parapsilosis, C. tropicalis, C. famata, and C. kefyr for fluconazole, itraconazole, voriconazole, caspofungin, posaconazole, and amphotericin B, during the two study periods. Error bars represent standard deviations. ${ }^{*} \mathrm{P} \leq 0.05$ by the two-way ANOVA test. 